\title{
An Image Highlights Removal Method with Polarization Principle
}

\author{
Li Fang ${ }^{1, ~ a ~}$ Tian Jiandong $^{2, b}$, Tang Yandong ${ }^{2, c}$ and Wang Yan ${ }^{1, d}$ \\ ${ }^{1}$ Shenyang Ligong University, Shenyang, Liaoning \\ ${ }^{2}$ State Key Laboratory of Robotics, Shenyang Institute of Automation, Chinese Academy of \\ Sciences, Shenyang, Liaoning \\ alifang061@163.com, ${ }^{b}$ tianjd@sia.cn, cytang@sia.cn, ${ }^{\mathrm{d}}$ wy2228@sohu.com
}

\begin{abstract}
Keywords: image processing; highlight removal; polarization information; Stokes parameters; specular reflection.
\end{abstract}

\begin{abstract}
The existing highlight detection and removal methods usually depend on color or some geometrical information. These methods cannot be used when highlight region degrades texture details of object surface. In this paper, we propose a novel, simple and effective method to solve this problem. In our method, we utilize dark channel to automatically detect highlight region. Then, based on polarization information of highlight region, specular refection component is removed. Our experimental results and comparison with other methods show that our method can effectively remove the highlight regions of the target image, and restore image texture details on the highlight regions under natural environment.
\end{abstract}

\section{Introduction}

Highlight region, generated by reflected light components in specular component, degrades texture details of object surface. The problem brings some difficulties to computer vision algorithms, such as segmentation, 3D reconstructions and object detection. To remove highlight, some approaches were proposed, and they usually includes two steps. The first step is to separate diffuse and specular components of surface reflection. The second one is to eliminate specular component and retain the diffuse component for an approximate specular-free image. According to the number of the images used for the removal of highlight regions, highlight removal methods can be roughly categorized into two categories: removal with single image and removal with multiple images.

For single image highlight removal, Tan and Ikeuchi [1] proposed an image decomposition method which is based on colors, particularly chromaticity, without explicit segmentation. Their approach achieves satisfactory results, however it fails in the presence of the colors which have the same hue component with different saturation one. In [2], Shen et al. introduced that the specular fractions of image pixels can be computed by intensity ratio. Using pixel clustering and intensity ratio sorting, the specular component of each pixel can be determined and removed. In [3], Kim et at al. demonstrated that for most natural images the dark channel can provide an approximate specular-free image. Then they utilize smooth prior on the specular reflection as well as the diffuse chromaticity in the dichromatic to refine the specular-free image. Yang et al. [4] [5] proposed an effective specular highlight removal method based on an observation that, in colour images, the maximum fraction of the diffuse colour component in diffuse local patches changes smoothly. Based on the observation, they developed a fast bilateral filtering approach to maximum chromaticity with neighboring pixels in real-time. The above mentioned methods [2] [3] [4] [5] usually fail under natural sunlight conditions for highlight removal.

For multi-image highlight removal, Stao and Ikeuchi [6] use multiple captured images under different directions of one light sources to separate diffuse and specular components. In order to remove highlights, the methods in [7] [8] need repeated change of light source directions, and do not work well in real applications. In [9], Umeyama et al. showed that specular component has a strong intensity and is polarized significantly compared to the diffuse component. They apply independent component analysis to some images obtained through a polarizer of different orientions, but it is 
difficult for them to manually choose the appropriate polarized orientions. Xu et al. [10] match two arbitrary images using SURF (Speeded Up Robust Features), and then minimize gray selection to eliminate the highlight and fuse relative regions. Because of image registration, their method is sensitive to illumination.

In this paper, we propose a novel highlight detection and removal method under natural sunlight conditions. First, a major highlight region of an image is automatically detected with dark channel prior. Then, based on information of the highlight region and our designed polarization control system, we obtain a precise polarizing angle to remove highlight.

This paper has three mainly contributions:

1) Our method can automatically detecte the highlight;

2) Our method has no particular requirements for object and environment, and can effectively remove the highlight regions under the natural illumination.

\section{Polarizer rotation control system}

Currently, almost all the polarizers in cameras are manually controlled. It is disadvantageous for cameras to adapt to environment, and it also brings large error to highlight removal results. For solving this problem, we design and realize an accurate control system for polarizer's angle.

\section{Automatic detection of highlight regions}

Dark channel image. Dark channel prior depends on the observation that in most of the non-sky outdoor haze-free images patches, at least one color channel has very low intensity at some pixels [11]. Highlight regions of non-sky image in three channels all have high intensity. However, nonhighlight regions of non-sky image must have one color channel with low intensity. According to the principle, we detect highlight region with dark channel prior.

A dark channel image of $I$ can be defined as follows:

$$
\begin{aligned}
I^{\text {dark }} & =\min _{\{r, g, b\}}\left\{m_{d} \Lambda+m_{s} \Gamma\right\} \\
& =\min _{\{r, g, b\}}\left\{m_{d}\left(\begin{array}{c}
\Lambda_{r} \\
\Lambda_{g} \\
\Lambda_{b}
\end{array}\right)+m_{s}\left(\begin{array}{c}
\Gamma_{r} \\
\Gamma_{g} \\
\Gamma_{b}
\end{array}\right)\right\} \\
& =\min _{\{r, g, b\}}\left\{m_{d}\left(\begin{array}{c}
\Lambda_{r} \\
\Lambda_{g} \\
\Lambda_{b}
\end{array}\right)\right\}+\min _{\{r, g, b\}}\left\{m_{s}\left(\begin{array}{c}
\Gamma_{r} \\
\Gamma_{g} \\
\Gamma_{b}
\end{array}\right)\right\} \\
& =\frac{1}{3} m_{s}
\end{aligned}
$$

Where $m_{d}$ and $m_{s}$ are the diffuse and specular reflection coefficients respectively, $\Lambda$ represent the chromaticities of the diffuse components. $\Gamma$ is the illumination chromaticity. Here, we normalize the illumination [2] and assume that the illumination is white. Fig.1 shows the highlight detection result of Shen [2] and ours. From the results, we can find that the method by dark channel to detect highlight is feasible. 




(a)

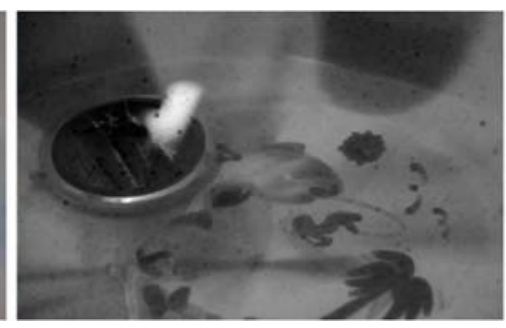

(b)

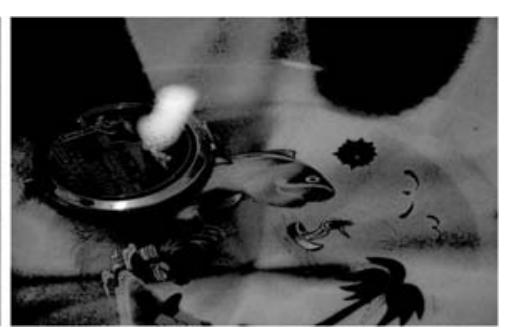

(c)

Fig.1. The experimental highlight detection results. (a) Original image; (b) Our method; (c) Shen [dark]

Obtain major highlight region. In order to obtain a precise angle of polarizer for highlight removal, and improve algorithm's efficiency, we only focus on the detection of the major highlight region. First, the dark channel image $I^{\text {dark }}$ is smoothed by the method in [12] to preserve major edge. The process is as follows:

$$
\begin{aligned}
& C(S)=\#\left\{p \| \partial_{x} S_{P}|+| \partial_{y} S_{p} \mid \neq 0\right\} . \\
& \min _{S}\left\{\sum_{p}\left(S_{p}-I_{p}^{\text {dark }}\right)^{2}+\delta \sqsubset C(S)\right\} .
\end{aligned}
$$

Where $I^{d a r k}$ and $S$ represent the in put image and the smoothed image. $\nabla S_{p}=\left(\partial_{x} S_{p}, \partial_{y} S_{p}\right)^{T}$ denotes the gradient for each pixel $p$ which is calculated as color difference between neighboring pixels along the $x$ and $y$ directions. Our gradient measure is expressed as $C(S) . \delta$ is a weight directly controlling the significance of $C(S)$, which is in fact a smoothing parameter. Finally, the smoothed image $I_{S}$ is segmented by Edge Detection and Image Segmentation System (EDISON) [13] to obtain major highlight region. This system has some parameters, such as feature bandwidth, spatial bandwidth and the minimum region area. In our experiments, we set spatial bandwidth is 10 and the minimum region area contains 200 pixels.

\section{Polarizer's angle for highlight removal [14]}

Measurement for polarization parameters. Specular component has a strong intensity and is polarized significantly compared to the diffuse component. Based on this principle, we firstly remove specular component with Stocks parameters.

Stokes parameter is used to represent the states of polarized light, and it is usually described as:

$$
S=\left[\begin{array}{llll}
S_{0} & S_{1} & S_{2} & S_{3}
\end{array}\right]^{T}
$$

Stokes parameter can be obtained by a linear relation:

$$
S_{\text {out }}=M \times S_{\text {in }} \text {. }
$$

Where $S_{\text {out }}, S_{\text {in }}$ and $M$ represent the output Stokes parameter, incident Stokes parameter and polarizing device, respectively. When any two of these three parameters are given, the unknown one can be obtained through (2).

$S_{i n}$ can be measured by the device in Fig.2. In this device, in order to compute $S_{i n}$, we need incident light $S_{\text {out }}$ and Mueller matrix $M$ in $R^{4 \times 4}$ representing material characteristics of polarizing film and phase delay timer. The Mueller matrix of the linear polarizer and a $1 / 4$ wavelength linear delay timer can be referred from [14]. Then we represent Stoke parameters as a $4 \mathrm{D}$ column vector in $\mathrm{x}-\mathrm{y}$ coordinates, meanwhile $\lambda$ is $0^{\circ}$, and $\beta$ equals $0^{\circ}, 45^{\circ}, 90^{\circ}$ and $-45^{\circ}$ separately. We can obtain Stoke parameters of incident light. 


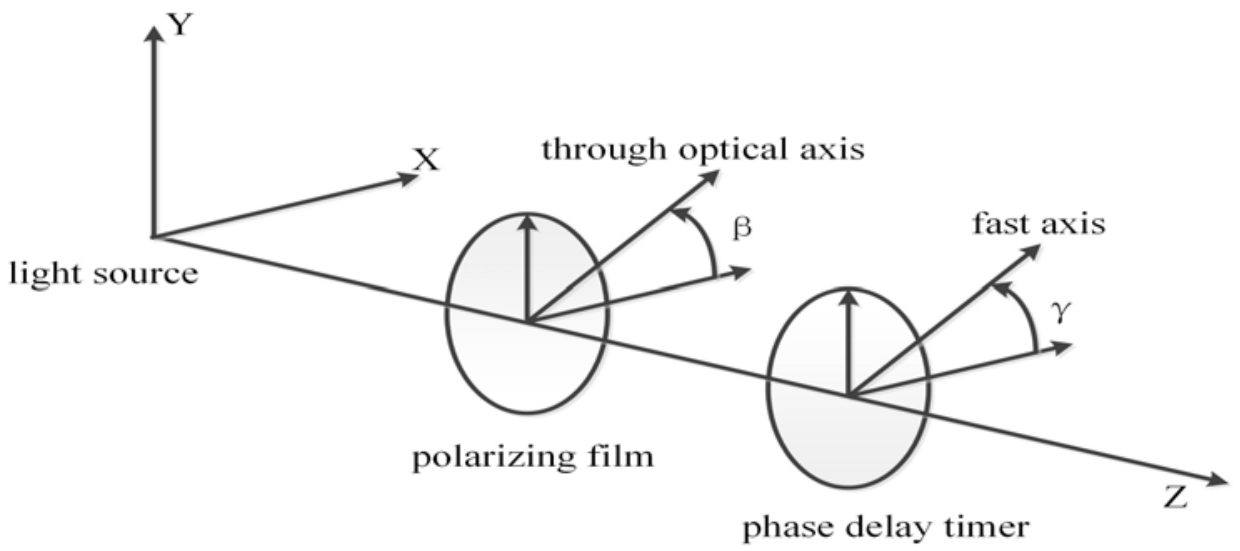

Fig.2. Design of the polarimeter

In real applications, since circularly polarized light rarely appears, we usually ignore $S_{3}$. Supposing that CRL is set up in front of the camera, and the pixel value $I(x, y, \theta)$ in $(x, y)$ depends on the polarizer's rotation angle $\theta[15,16]$, can be represented as:

$$
I(x, y, \theta)=\frac{1}{2} S_{0}(x, y)+\frac{1}{2} S_{1}(x, y) \cos 2 \theta+\frac{1}{2} S_{2}(x, y) \sin 2 \theta .
$$

Here, $S_{0}, S_{1}$ and $S_{2}$ represent the first three Stokes parameters[17]。

The precise rotation angle for highlight removal. Based on the selected major highlight regions and Stokes parameters, Eq. (5) can be used to obtain the precise rotation angle for highlight removal. Finally, the precise angle $\theta$ for highlight removal can be optimized to minimize $I_{c}(x, y, \theta)$.

$$
\theta_{p}=\underset{\theta}{\arg \min } I_{c}(\mathrm{x}, \mathrm{y}, \theta), \quad \theta=0^{0}, 1^{0}, 2^{0} \ldots \ldots .180^{\circ} .
$$

\section{Experiments and Discussion}

In order to evaluate the performance of our approach, we took some pictures with Canon60D camera. Unlike the methods [4], we need no particular requirement for the experimental objects, light sources, and types of images. All of our experimental pictures were automatically taken by our designed polarizer rotation control system under the natural illumination.
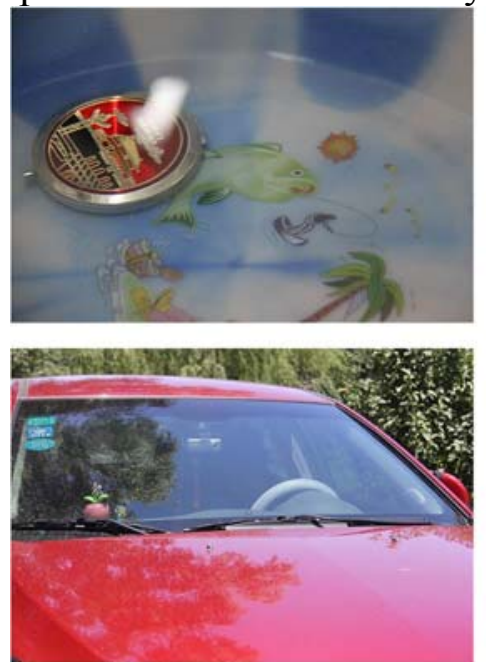

(a)
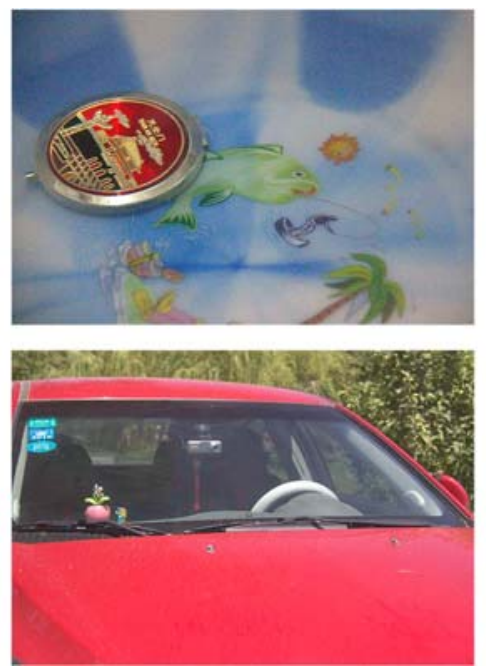

(b)
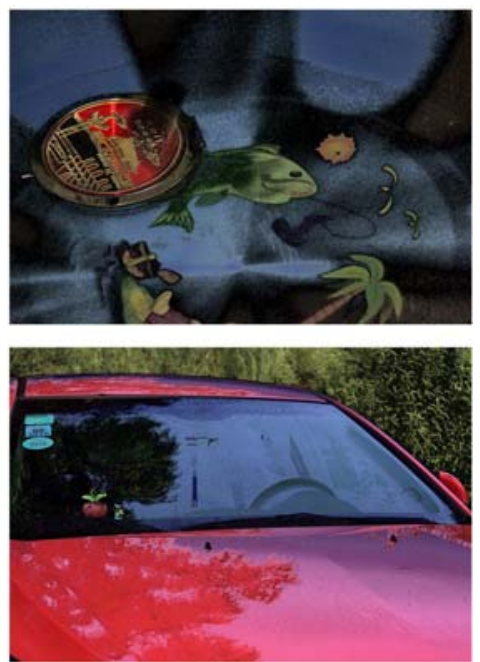

(c)

Fig.3. Comparisons of different methods' results. (a) Original image; (b) our method; (c) Yang [4]; 
In Fig.3, from the results, we can find that the methods in [4] fail to tackle the highlight removal task. The highlight removal results except ours not only have biased color, but also degrade the details of the original image. Its reason is maybe that almost all other highlight removal approaches require some specific imaging conditions, such as light sources, and types of images.

\section{Acknowledgement}

In this paper, the research is sponsored by the Nature Science Foundation of Shenyang Province (Project No. 61473280).

\section{References}

[1]R T Tan and K Ikeuchi, Separating Reflection Components of Textured Surfaces Using a Single Image [J], IEEE Transactions on Pattern Analysis and Machine Intelligence, 2005, 27(2): 178-193.

[2]Shen H L, Zheng Z H. Real-time highlight removal using intensity ratio. Applied optics, 2013, 52(19): 4483-4493.

[3]Kim H, Jin H, Hadap S, et al. Specular Reflection Separation Using Dark Channel Prior[C]// Proceedings / CVPR, IEEE Computer Society Conference on Computer Vision and Pattern Recognition. IEEE Computer Society Conference on Computer Vision and Pattern Recognition2013:1460-1467.

[4]Q Yang, S Wang, N Ahuja. Real-time specular highlight removal using bilateral filtering [M]//Computer Vision-ECCV 2010.Springer Berlin Heidelberg, 2010: 87-100.

[5]Q Yang, J Tang, N Ahuja. Efficient and Robust Specular Highlight Removal [J]. IEEE Transactions on Pattern Analysis \& Machine Intelligence, 2015, (1):1-1.

[6]Y Sato and K Ikeuchi. Temporal-color space analysis of reflection [J]. Journal of the Optical Society of America A, 1994,11(11): 2990-3002.

[7]S Lin, H Y Shum. Separation of diffuse and specular reflection in color images[C]/Proceedings of the IEEE International Conference on Computer Vision and Pattern Recognition(CVPR'01),Kauai, USA, $2001,(1): 341-346$.

[8]S Lin ,Y Li, S B Kang, X Tong and H Y Shum, Diffuse-specular separation and depth recovery from image sequence[M] ]//Computer Vision-ECCV 2002. Springer Berlin Heidelberg, 2002: 210-224.

[9]S Umeyama, G Godin. Separation of diffuse and specular components of surface reflection by use of polarization and statistical analysis of images [J]. IEEE Transactions on Pattern Analysis and Machine Intelligence, 2004, 26(5):639-647.

[10]Xu Y, Wang F, Zhao Y. Matching based Highlight Removal[C]// Multimedia Technology (ICMT), 2010 International Conference on IEEE, 2010:1 - 4.

$[11]$ K. He, J. Sun, and X. Tang. Single image haze removal using dark channel prior. IEEE Transactions on Pattern Analysis and Machine Intelligence. 33(12):2341-2353,2011.

[12] Xu L, Lu C, Xu Y, et al. Image Smoothing via L0 Gradient Minimization[J]. Acm Transactions on Graphics, 2011, 30(6):61-64.

[13] http://coewww.rutgers.edu/riul/research/code/EDISON/doc/overview.html

[14] Bhaskar K, Shyama R, Hem Chandra K. Direct determination of the generalized Stokes parameters from the usual Stokes parameters [J]. Optics Letters, 2009, 34(6):719-721. 
[15]M W Hyde, S C Cain, J D Schmidt, et al. Material classification of an unknown object using turbulence-degraded polarimetric imagery [J]. IEEE Transactions on Geoscience and Remote Sensing, 2011, 49(1):264-276.

[16]K M Yemelyanov, J A McVay, N Engheta, et al. Adaptive polarization-difference imaging algorithms for through-the-wall microwave imaging scenarios [J]. 2005.

[17]S Fang, X S Xia, H Xing, et al. Image dehazing using polarization effects of objects and airlight [J]. Optics express, 2014, 22(16): 19523-19537. 